\title{
Treatment of Late Menstruation from "Chong Mai Li Yang Ming”
}

\author{
Yuting Bo, Zhefeng Liu* \\ Shaanxi University of Traditional Chinese Medicine, Xianyang 712000, Shaanxi, China.
}

\begin{abstract}
How to cite this paper: Yuting Bo, Zhefeng Liu. (2020) Treatment of Late Menstruation from "Chong Mai Li Yang Ming”. International Journal of Clinical and Experimental Medicine Research, 4(4), 236-241.

DOI: $10.26855 /$ ijcemr.2020.10.019
\end{abstract}

Received: October 13, 2020

Accepted: November 8, 2020

Published: November 16, 2020

*Corresponding author: Zhefeng Liu, Shaanxi University of Traditional Chinese Medicine, Xianyang 712000, Shaanxi, China.

\begin{abstract}
Late menstruation is one of the common menstrual diseases. Its etiology and pathogenesis are complex and closely related to the five Zang Fu organs and Chong Ren. Chong pulse is one of the Eight Extraordinary Meridians, the sea of five viscera and six Fu organs and blood sea. It is the basis of menstruation and an important factor for the normal menstruation. "Chong Mai is subordinate to Yangming", and Yangming is the sea of water and valley, and the source of Qi and blood transformation is the main source of Chong Ren's blood. When Yangming is prosperous, the Chong pulse is full, and the tide can come on time through water. Therefore, it is of great significance to treat late menstruation from Yangming. From the relationship between Chong Mai, Yangming and late menstruation, the author discusses the importance of treating late menstruation from Yangming, and provides more theoretical support and clinical reference for the treatment of this disease from the perspective of "Yangming".
\end{abstract}

\section{Keywords}

Later Period of Menstruation, Chongmai is Subordinate to Yangming, Yangming, Chongmai

Late menstruation refers to the postponing of menstrual cycle for more than 7 days, even 3-5 months, and continuous occurrence of more than 2 cycles is called "late menstruation", or accompanied by menstrual volume and menstrual abnormalities and other symptoms, serious can develop into amenorrhea or even infertility, is a common clinical menstrual disease, also known as "water expired", "late", "late menstruation", "to the period cannot come". It is the main symptom of premature ovarian failure, polycystic ovary syndrome, infertility and other diseases. In the treatment of Western medicine, estrogen and progesterone sequential therapy is commonly used to induce ovulation and adjust menstrual cycle, but hormone drugs have many side effects and long-term effect is not good, so it is not suitable for long-term use. Traditional Chinese medicine (TCM) is based on the principle of holistic concept and syndrome differentiation and treatment. It has rich experience and achieved good therapeutic effect in clinic. This disease was first recorded in Zhang Zhongjing's synopsis of the Golden Chamber - treatment of women's miscellaneous diseases with pulse and syndrome: "Wenjing decoction can't treat menstrual flow to the end". "Su Wen - ancient innocence theory" says: "on February 7 and the day is right, Ren Maitong, Taichong maisheng, monthly affairs with the current..." "Women's regulations" also said: "the essence of menstruation lies in the Chong pulse, the stomach qi, and the source ear of the heart and spleen.” It can be seen that ancient doctors believed that the pulse was the root of menstruation, and it was an important factor in whether menstruation could come on time. But "Chong Mai is subordinate to Yangming", which is closely related to physiology and pathology. Yangming is the sea of water and valley and the source of Qi and blood transformation. Only when the function of spleen and stomach is normal, and the source of blood and blood of Yangming is sufficient, can it be injected into chongmai 
palace. Therefore, the normal physiological function of the spleen and stomach affects the rise and fall of the Chong pulse Qi and blood, which leads to the occurrence of menstrual diseases.

\section{Etiology and pathogenesis of late menstruation}

The earliest record on the etiology and pathogenesis of late menstruation is Su Wen: "the heart and spleen are caused by two Yang diseases, and there is no hidden Qu, and women are not in the moon". It is proposed that internal injury of seven emotions can cause menstruation to come out of time [1]. The spleen and stomach are the source of Qi and blood. The liver stores blood and the kidney stores essence. The essence and blood are homologous. Women take blood as the basis. Therefore, the occurrence of this disease is closely related to the liver, spleen and kidney. "Zhulin women's Department of syndrome and treatment" divides the menstrual delay into several types, such as overdue menstruation, several months menstruation, body fat overdue menstruation and thin overdue menstrual line [2]. "There is no other disease in leptin, and the Qi and blood is insufficient for those who have expired". "If you eat too much fat, and if you pass the time limit, the dampness phlegm will be stagnant and the body fat will be forced". "Chen su'an gynecology tonic explanation" said: "the woman after the late water, blood deficiency, this is due to spleen and stomach weakness, diet reduction, cannot produce blood". The material basis of menstruation is blood, and the water and valley transformed by the spleen and stomach is the main source of Qi and blood. If the physiological function of the spleen and stomach is abnormal, one is that the essence and blood metaplasia is passive and can't fill the uterus on time, resulting in the wrong menstruation; the second is that phlegm dampness is endogenous, phlegm and evil Qi hinder the circulation of Qi and blood, and the blood flow is not smooth, which leads to the late period of menstruation. "Jing Yue Quan Shu women’s regulations" says: "Yin Fire flickers, blood is hot, and every expired, this water is less blood deficit, dry and astringent". "If blood is cold, it will come in the later period of menstruation. However, if the blood is cold and Yang Qi is insufficient, then cold comes from it, and biochemical loss is the so-called cold”. It can be seen that blood cold and blood heat are also the important pathogenesis of menstrual errors. Generally speaking, the pathogenesis of late menstruation can be divided into deficiency and excess. For those with deficiency, Qi and blood are deficient, and there is no source of meridian blood. Therefore, the deficiency of Chong pulse results from the late stage of menstruation; for those with excess, external cold and dampness evil, or phlegm dampness block, hinder the circulation of Qi and blood of meridians, and the blood circulation of Chong pulse is not smooth, Qi and blood cannot reach the uterus on time, and the menstrual blood will not come.

\section{Theoretical basis of “Chong Mai Li Yang Ming”}

The theory of "Chong Mai is subordinate to Yangming" originated from Neijing: "Chong Mai is the sea of meridians, which mainly permeates and irrigates valleys, with Yangming and Yu zongjin". However, this theory was formally put forward in the compendium of Jiyin Tiaojing gate in the Ming Dynasty [3]: the Yin blood of Director Ren Mai. Taichong belongs to Yangming, the sea of blood. Therefore, if the valley is full of Qi, then the sea of blood will be full, and the moon will be under the current situation. "This shows that there is a close relationship between chongmai and Yangming. From the circulation and distribution of meridians, Su Wen Gu Kong Lun No.60 says, “Chong Mai originates from Qi street The chivalrous navel goes up to the chest and disperses". "And the stomach is a sea of rivers and valleys. Its stomach gas transportation in the gas street. That is, air burst Yangming and chongmai intersect in Qi street. In terms of physiological function, Chong is the sea of blood and the sea of twelve meridians. Chong pulse can regulate qi and blood and is the channel of Qi and blood movement. Zhang Jingyue said: "Chong is the sea of five viscera and six Fu organs, and the blood of viscera belongs to Chong pulse". Yangming is a meridians of many Qi and blood. Yangming's stomach meridian receives rotten water and grain. For example, it is noted in Lei Jing that "two Yang, Yang Ming are the meridians of stomach and large intestine Husband's stomach is the sea of water and valley, which dominates the operation and defense of the army and moistens the tendons of the clan The essence and blood flow is the foundation of Biochemistry, but Yangming is the most important "Women's regulations" says: "and Yangming stomach qi is the root of Chong pulse". Luo guogang: "the rise and fall of blood and Qi stems from the rise and fall of shuigu, and Yangming stomach meridian is the root of Chong pulse". The spleen and stomach are the source of Qi and blood biochemistry, and the blood of Chong pulse comes from the essence of water and grain transformed by Yangming. Only when the function of Yangming stomach meridian is normal and the Qi and blood is vigorous, can the Chong pulse function normally. Such as "on blood syndrome": "the treatment of Yang Ming is the treatment of Chong". 


\section{Pulse and late menstruation}

Chong is the sea of blood and the sea of five viscera and six Fu organs. It is one of the eight channels of Qi and blood circulation. It is the root of menstruation. When the blood is filled with blood, it can be injected into the uterus to keep up with the times. Such as "plain questions ancient innocence theory" said: "on February 7 and the day is GUI to, Ren Mai Tong, Taichong pulse Sheng, monthly affairs with the current...”. "Jing Yue Quan Shu women’s regulations" said: "the Yin blood is the origin of menstruation, and there is no internal organs? Only the blood of Zang Fu organs belongs to Chong pulse, which is the blood sea of five viscera and six Fu organs. Therefore, it is said in the Scripture that Chong pulse is abundant, and the moon is in the current situation". "Nvke Jinglun" said: "women's monthly affairs, the original Taichong pulse Sheng, and Chong pulse from the cell, that is, the sea of blood, the source of water". These fully show that Chong pulse is the basis of menstruation. Women take blood as the foundation, while Chong pulse is the sea of blood. If Chong Mai loses its nourishment, the water will not be adjusted. As Xu Lingtai of Qing Dynasty said: "to treat a woman, you must first know Chong Ren Mai”. Chong pulse is not only closely related to the generation and operation of Qi and blood, but also to Yangming stomach meridian, Jueyin liver meridian and Shaoyin kidney meridian in meridian transport. It can be seen that Chong pulse is an important factor in whether menstruation can come on time. Therefore, in clinical treatment of late menstruation, starting from the deficiency and excess of Chong pulse, good clinical effect can be obtained [4].

\section{Yangming and late menstruation}

"Nvke Jinglun” said: "two Yang, foot Yang Ming stomach pulse. He is the official of Cang, who is in charge of water and valley, but cannot accept it. This is from the heart and spleen. Because of the fact that women can't hide and bend, the heart can't produce blood, and blood can't nourish the spleen. At first, the stomach can't receive it, and then the spleen can't be accepted. Therefore, stomach disease occurs in the heart and spleen. Because of the decline of water and grain, there is no way to change the essence of Qi, then the blood vessels will wither, and the moon cannot go down". "Clinical Guide to medical records" said: "where the blood to the beginning of the chongmai, this pulse of stomach by the tube". Thus, the normal menstrual flow and the occurrence of menstrual diseases are closely related to Yangming. Yangming is a scripture of much Qi and blood. "Plain questions-explanation of Yangming pulse" says: "Yangming is the stomach pulse, and the stomach is the earth Yang Ming dominates the flesh, and its pulse is full of blood and Qi”. Yangming is not only the stomach meridian, but also the whole system of the spleen and stomach transporting water and grain essence to the viscera [5]. If Yangming is too easy to change heat, dry heat injures the body fluid, and the body fluid is insufficient, the blood sea cannot be filled, and the Chong pulse and the uterus lose the ability to nourish. Cheng Ruoshui said: "women's menstrual blood is transformed by the essence and Qi of five tastes of water and grain”. The Qi of Yang Ming is the spleen in the viscera and the stomach in the Fu organs. The spleen and stomach belong to the middle energizer, which are the source of Qi and blood biochemistry and the sea of five viscera and six Fu organs. "Taisu of Huangdi’s Internal Classic says: "the moon does not come, and the disease is due to the stomach". Therefore, if the physiological function of Yangming is normal, then Qi and blood are vigorous, Chong Ren can be fully fed, and menstruation can be on time. If the diet is not proper, the spleen and stomach function is out of balance, the stomach is accepted and rotten, the water and grain are abnormal, and the Qi and blood are metabolized without a source, resulting in deficiency of Qi and blood, then the Chong pulse Qi and blood are also deficient, the blood sea cannot be filled, and the menstrual water cannot come in time. The spleen and stomach belong to the middle Jiao, which is the hinge of Qi rising and descending. Huang Yuany u said: "in the later stage, the stagnation of wood Qi is controlled, and the mechanism of closing the knot is also. Its original total due to spleen dampness and liver depression. Wood qi depression depression, not to carry forward, then blood coagulation it can be seen that spleen deficiency dampness leads to poor liver qi, hinders the normal operation of Qi and blood. "Chong Mai is attached to Yangming", which is closely related to menstruation and menstrual diseases. Therefore, we can treat the late menstruation from the perspective of Yangming.

\section{On the treatment of late menstruation from Yang Ming}

Modern physician Chai Songyan's academic thought of “two Yang pathogenic” makes it clear that women's Yangming lesions can also lead to menstrual diseases [6]. The causes of Yangming disease are various, such as deficiency of Qi caused by dry heat of Yangming, imbalance of qi circulation and descending, deficiency of Yangming Qi, etc. [7], These are closely related to the function of spleen and stomach, and the dysfunction of spleen and stomach is an important link leading to Yangming disease. 


\subsection{Tonifying Yang Ming Jin Qi}

Yangming is a meridians with much Qi and blood. If the ordinary diet is not regular, he likes to eat spicy and thick Houji products; if his emotions are not smooth, the five emotions are too polarized; or feel the warm and evil Qi, which leads to Yang Ming dryness and heat, the stomach likes moistening and evil dryness, and the dry heat consumes the body fluid, resulting in Yang Ming deficiency and deficiency of stomach yin [8]. Yang Ming is dry and hot, which consumes the body fluid and Qi, but the blood and the blood are of the same origin. The blood sea cannot be filled due to the deficiency of the body fluid. The Chong pulse and the uterus lose the ability to nourish, resulting in the later period of the moon water. In addition to the main symptoms of irregular menstruation, the common accompanying symptoms, such as thirst and drinking, dry stool and so on. The treatment should nourish yin and clear stomach, such as "Yizong Jinjian deleting and reinforcing famous medical prescription theory" said: "Yangming stomach has more Qi and blood, and the two Yang combined with Ming is hot, which is to enter into the disease often solid If you drink alcohol and fat, and burn it too much, so that dampness and heat are blocked in the stomach and against the meridians and collaterals, it is a disease. If it hurts the blood, it should be treated to clear the stomach”. Tonify Yang Ming Jin Qi, so that the Chong pulse can be fed, Chong pulse blood sea overflowing, then by regulating. Liu Feng, a famous old Chinese medicine doctor in Beijing, is good at treating Yangming. He believes that reducing Yang Ming is due to the reduction of Chong Qi, and tonifying yang ming (nourishing stomach yin) is enough to benefit Chong pulse. Clearing Yang Ming can cool blood and regulate Chong Ren. The representative prescription is Guashi decoction [Fructus Trichosanthes 15g, Dendrobium 12g, Shengdi 12g, motherwort 9g, Coptis 6G, Scrophularia 9g, plantaginis 9g (package), Achyranthes bidentata 12g, Qumai 12g, Maidong 9g]. This prescription can clear the heat and dryness of Yangming, increase fluid and produce fluid to nourish the source of no root. It has good effect on amenorrhea, late menstruation and less menstruation caused by Yangming dryness and heat [9].

\subsection{Flat thrust and downthrust}

Zhang Xichun said, “Chong is a sea of blood and lives on both sides of Shao's abdomen. Its pulse Li Yang Ming, lower even Shaoyin Yang Ming stomach deficiency, its Qi cannot be Zhenan Chong Qi, Chong Qi is also easy to dry "Plain questions-Gu Kong Lun" that "Chong pulse for disease, against the Qi in the urgent". Chongmai is attached to Yangming [10]. When Yangming is deficient in stomach and unable to absorb Chong Qi, Chong Mai Qi goes against it [11]. The treatment should be tonifying spleen and stomach, regulating Chong and descending adverse. Chong pulse Qi and blood harmony, menstruation with the current. Li Dongyuan mainly used Tiaozhong Yiqi Decoction. Professor Zhou Mingxin [12] it is commonly used to remove ochre, Gastrodia elata, Inula, areca nut and Muxiang to promote qi descending Chong; ginger, pinellia and weijiangchong.

\subsection{Nourishing blood and regulating menstruation}

The spleen and stomach are the foundation of the postnatal. They are in the same position in the middle Jiao, and they are the source of Qi and blood. Wu Shuqing: "if a woman's menstrual blood does not regulate, she must examine the source of her temper transforming into health, and strengthening the spleen is the key to regulating menstruation". If the ordinary weak, or plain diet misconduct, like eating cold things, damage the spleen and stomach, spleen and stomach transport dysfunction, unable to transport water grain, blood essence; or excessive diet in order to lose weight, Qi and blood biochemical passivity; resulting in deficiency of Qi and blood, Chong pulse blood cannot overflow on time, such as "bamboo forest female division syndrome treatment": "leptin has no other disease, and expired by the Qi and blood is insufficient”. In the treatment of deficiency of Qi and blood in spleen and stomach, it is necessary to replenish qi and nourish blood. The prescription is composed of Siwu Decoction, Sijunzi Decoction and motherwort. Sijunzi Decoction can invigorate spleen and Qi, Siwu Decoction can nourish blood and regulate menstruation, and then motherwort can activate blood and regulate menstruation. Modern doctors use Bazhen Yimu decoction as the main addition and subtraction (to roast Astragalus membranaceus, Poria cocos, Caulis Spatholobus, Lycium barbarum, black sesame, Ligustrum lucidum, jujube, safflower) self-made Buqi Yangxue recipe, and achieved good clinical effect [13].

\subsection{Invigorate spleen and soothe liver}

"Clinical guide medical records" says: "Taiyin wet soil, get Yang before transport”, Ruoyu likes to eat fat, sweet and greasy, hurt the spleen and stomach, spleen and stomach is not healthy, phlegm dampness endogenous, dampness dampness block Qi, Chong pulse Qi and blood flow is not smooth, resulting in menstrual error. For example, 
in the bamboo grove women's department of syndrome and treatment: "those who have too much fat diet but have expired will have dampness phlegm stagnation and body fat pressure". Danxi Xinfa: "if a fat woman is rich, she will be indulged in eating and drinking, and her menstrual flow will not be adjusted". Appropriate line wet dry phlegm, with Daotan soup and so on Wan Quan of Ming Dynasty advocated Banxia Fuling pill, Kaiyu Erchen decoction, Liujunzi plus guixiong decoction, etc. Fu Qing mainly treats dampness and does not forget to soothe the liver. He is good at using Bupleurum to regulate liver qi and prevent earth blocking and liver depression [14]. Huang Yuanyu thinks that the reason of the later period of menstruation is the disorder of liver qi, and the root of liver depression is the disorder of qi movement caused by spleen and stomach deficiency. "Jiangling Ejiao Decoction" was selected by Huang's late treatment of menstrual water, which was composed of Guizhi Jiangling decoction, Paeonia lactiflora, donkey hide gelatin and Salvia miltiorrhiza. It has the effects of invigorating spleen and nourishing blood, soothing liver and activating blood circulation [15].

\subsection{Tongjiang stomach qi}

"Menstruation with the current”, not only to flush the blood sea overflowing, but also need to Chong pulse blood downward. Such as ye Tianshi said: "where the water to, by the Chong pulse and the beginning of the next, this pulse of stomach by the tube". "Su Wen Gu Kong Lun No.60": "Chong Mai, originated from Qi street The chivalrous navel goes up to the chest and disperses". It can be seen that the Qi and blood of Chong pulse is ascending, and it can only go down by relying on Yangming Meridian. Therefore, only when the circulation of Yangming is normal, can the blood and blood of Chong pulse run smoothly [16], menstrual blood can go down on time. If the stomach qi does not fall, it will affect the operation of Qi and blood, resulting in delayed menstruation. For example, "regulating menstruation and nourishing blood should not be given priority to SHUNQI first", "regulating menstruation should not be prior to nourishing blood, and nourishing blood should not be prior to regulating qi". According to the clinical symptoms, some doctors think that the causes of Stomach Qi not falling are stomach heat and spleen deficiency, stomach and intestine heat and stomach qi stagnation. For those with stomach heat and spleen deficiency, Banxia Xiexin Decoction should be selected; for those with stomach and stomach heat, pungent and bitter herbs should be opened and bitter to reduce, and fullness should be eliminated. Rhubarb Huanglian Xiexin Decoction should be used for stomach qi stagnation, and Zhizhu pills should be used for reducing qi stagnation [17].

\section{Examples of proven cases}

The patient, a 26 year old female, was first diagnosed on August 10, 2019. Chief complaint: delayed menstruation with less than one year. Nearly one year, menstrual cycle 60-90 days, menstrual period 5 days, less amount, dark red color, blood clot, menstrual abdominal distension pain, premenstrual breast distension pain. LMP: 2019-06-12. HCG was negative in urine. Usually chest tightness and discomfort, easy fatigue, poor food intake, poor night sleep, loose stool when dry, light tongue moss white greasy, tongue with tooth marks, pulse string weak. Gynecological ultrasound showed that the thickness of intima was $0.8 \mathrm{~cm}$. TCM diagnosis: late menstruation; western medicine diagnosis: sparse menstruation. Dialectical: spleen deficiency and liver depression. Treatment: strengthening spleen and nourishing blood, soothing liver and activating blood circulation. Fangxuan Xiaoyao Powder addition and subtraction; drug composition: Danggui 15g, Chishao 10g, bupleurum 10g, Poria cocos 15g, dangshen 10g, fried Atractylodes macrocephala 15g, stir fried yam 12g, Shudi 15g, Chuanxiong 10g, Danshen 15g, Honghua 12g, Guizhi 10g, Ejiao 10g (closing), Amomum villosum 6G, dried ginger 3G, roasted licorice 6G, 7 doses in water.

Second visit on August 17: She complained of menstruation after taking 5 doses of medicine, with dark red color, moderate amount and blood clot. Then add $20 \mathrm{~g}$ motherwort to the original prescription. After 3 cycles of treatment, the menstrual cycle returned to normal.

Note: the patient is usually depressed and likes to eat fat and sweet food. For a long time, it damages the spleen and stomach. The spleen loses its healthy movement and produces phlegm dampness. The dampness hinders the qi movement. On the one hand, the blood circulation of the Chong pulse is not smooth, and the blood sea of the chongmai palace cannot be overflowed on time; on the other hand, the liver Qi is not dredged smoothly. At the same time, the spleen and stomach are weak, Qi and blood biochemistry are passive, can't nourish the Chong pulse and the cytoplasmic palace, the cell vein is empty, there is no menstrual blood to go down, so the late menstruation. Chong Mai is the basis of menstruation, and "Chong Mai is subordinate to Yangming". The blood of Chong Mai depends on the refined blood of water and grain transformed by Yangming, that is, the function of Yangming is normal, the essence blood is transformed into active energy, and the Chong meridian is nurtured. If the method of invigorating the spleen and activating blood, soothing the liver and activating blood is the therapeutic method, 
Xiaoyao Powder is selected. By tonifying Yangming, Qi and blood are active, and the Chong pulse Qi and blood are regulated smoothly, then it will arrive on schedule.

\section{Epilogue}

Generally speaking, the pathogenesis of late menstruation can be divided into two types: deficiency of Qi and blood, deficiency of menstrual blood, no source of menstrual blood, unable to overflow on time; in the case of excess, evil Qi hinders Qi, causing blood stasis over time, and the sea of Chong pulse and blood cannot overflow on time, so the menstrual period is wrong. Women with blood as the basis, Chong for blood sea, menstruation, Chong pulse imbalance is the woman's menstrual water. Yangming is a meridian with many Qi and blood, which is the source of Qi and blood transformation. "Chong Mai Li” and "Yangming” are the source of Qi and blood. The filling of Chong Mai Xue Hai depends on the spleen and stomach to transport the essence of water and valley. Therefore, Yangming function is normal, Qi and blood are full, and the Chong pulse and Blood Sea overflows on time, then the channels are regulated smoothly. If the physiological function of Yangming is abnormal, which affects the generation of Qi and blood and the operation of Qi, the sea of blood cannot overflow on time, leading to the late lunar water. "Treatise on blood syndrome" states that "the treatment of Yang Ming is the treatment of Chong". Through tonifying Yangming, on the one hand, Qi and blood are active, the Chong pulse can be nurtured, and the menstrual blood can be active; the second is to regulate the rise and fall of Qi, and the blood can reach the uterus on time, and menstruation will come in time. Therefore, it is of great significance to treat Yang Ming in the later period of menstruation.

\section{References}

[1] Yu Yingshi. (2019). Meta analysis of traditional Chinese medicine in the treatment of late menstruation [D]. Nanjing: Nanjing University of Traditional Chinese Medicine.

[2] Zhang Mingming, Wang Fenglan. (2016). Preliminary study on menstrual cycle abnormality in zhulinsi women's Department of syndrome and treatment [J]. Chinese Journal of Traditional Chinese Medicine Library and Information, 2016, 40(4): 56-58.

[3] Yan long, Xu Xin. (2019). Literature study on the relationship between Chong pulse and Yangming in Ming Dynasty [J]. Global Traditional Chinese Medicine, 2019, 12(5): 708-712.

[4] Xu Chongming, Wang Ziyuan. (2007). Treatment of irregular menstruation from Chong pulse [J]. Chinese Journal of Traditional Chinese Medicine, 2007, 25(10): 2033.

[5] Zhang Yingjie, Sui Hyatt. (2019). On the correlation between Yangming and five zang organs and female reproductive physiology [J]. Shandong Journal of Traditional Chinese Medicine, 2019, 38(9): 817-820.

[6] Teng Xiuxiang, Pu Lingyun. (2015). Analysis of Chai Songyan's academic thought and clinical experience of "eryang pathogenic disease” [J]. Chinese Medicine Information, 2015, 32(1): 65-66.

[7] Pan Xia. (2008). Based on the theory of "two Yang diseases occur in the heart and spleen" in Neijing, to explore the treatment of "women without moon" [D]. Chengdu: Chengdu University of Traditional Chinese Medicine.

[8] Xu zexia, Li Jun. (2014). Preliminary study on the mechanism of tonifying yang ming method in the treatment of menstrual diseases [J]. Sichuan Traditional Chinese Medicine, 2014, 32(4): 50-51.

[9] Pan Jinli. (2008). Guashi Decoction in the treatment of 40 cases of amenorrhea [J]. Chinese Medicine Research, 2008, 21(6): 57-58.

[10] Lin Qijia. (2011). Ancient literature research on Chong Mai [D]. Beijing: Beijing University of Traditional Chinese Medicine.

[11] Zeng Jihong. (2016). Analysis of the relationship between Chong Qi upwardness and late menstruation [J]. Modern Diagnosis and Treatment, 2016, 27(22): 4244-4245.

[12] Liu Guantao. (2014). Affinity reagent of traditional Chinese medicine [M]. Beijing: Xueyuan Publishing House, $2014,78$.

[13] Yao Tingzhou, Wan Zhiju. (2019). Observation on the curative effect of Qi and blood nourishing therapy in the treatment of late menstruation of Qi and blood deficiency type [J]. Guangxi Traditional Chinese medicine, 2019, 42(4): 27-29.

[14] Wang Lei. (2015). Theoretical analysis on the treatment of polycystic ovary syndrome from phlegm dampness [D]. Henan, Henan University of Traditional Chinese Medicine, 2015.

[15] Tian Huan, Wang Sheng. (2014). Huang Yuan’s treatment of menstrual diseases with Guizhi and poria cocos [J]. Jiangsu traditional Chinese Medicine, 2014, 46(3): 67-68.

[16] Fang Chuanming, Xie Chunguang. (2018). Treatment of perimenopausal syndrome based on "Yang Ming is closed” [J]. Chinese Journal of Basic Medicine of Traditional Chinese Medicine, 2018, 24(5): 705-706.

[17] Liu Xinmin, Zhao Yiming. (2014). The role of reducing stomach qi in the treatment of polycystic ovary syndrome [J]. Global Traditional Chinese Medicine, 2014, 7(5): 357-358. 